HERITABILITY OF HUMAN FUNCTIONAL ADAPTABILITY

V. KLISSOURAS, M.D.

Ergophysiology Laboratory, McGill University, Montreal, Canada

\begin{abstract}
The use of co-twin analysis in this laboratory has shown that Functional Adaptability (FA) is set by the genotype, although it can be substantially modified by training.

The present experiments are an extension of our earlier work and were conducted to establish quantitatively the heritability of FA in man.

Heritability refers to the proportion of the variation in FA which is attributable to genetic predisposition, while FA refers to the upper limit of adaptional response of the organism to a metabolic stressor, and is characterised by the Maximal Aerobic Power (MAP) and Maximal Anaerobic Capacity (MAC).
\end{abstract}

One hundred Monozygotic $(\mathrm{MZ})$ and Dizygotic (DZ) twins ran to exhaustion on progressively increasing work loads on the motor driven treadmill, in order to determine their MAP, MAC and maximal cardiac output. Their zygosity was diagnosed on the basis of morphology, dermatoglyphics and blood groups.

The contribution of heritability to FA was disclosed by variance analysis and by the equation:

$$
H=\frac{{ }^{6} D^{2}-{ }^{6} M^{2}}{{ }^{6} D^{2}}
$$

where $H=$ Heritability; ${ }^{6} D^{2}=$ variance of $F A$ within $D Z$ twins and ${ }^{6} M^{2}=$ variance of $F A$ within $M Z$ twins. Findings $\rightleftharpoons$ were discussed in relation to trainability and specific attributes which limit functional adaptability.

\title{
THEORY OF CONTROL AND MODEL APPLICATION IN THE STUDY OF PHYSIOLOGY OF SPORTS AND TRAINING
}

C. MOUTZITHROPOULOS, M.D.

Director of Bibliotheca Cardiologica, 6 Asklipiou Street, Athens, Greece

\begin{abstract}
Proposals are presented for a number of mathematical models of the various physiological systems which may prove useful in obtaining and integrating data about the functioning of these systems, especially the cardiovascular and locomotor ones.
\end{abstract}

